\title{
Biología reproductiva y conservación: el caso de la regeneración de bosques templados y subtropicales de robles (Quercus spp.)
}

\author{
Plant reproductive biology and conservation: the case of temperate and subtropical \\ oak forest regeneration
}

FERNANDO J. PULIDO

Departamento de Biología y Producción de los Vegetales, Escuela de Ingeniería Técnica Forestal, Centro Universitario, Universidad de Extremadura, 10600 Plasencia, Cáceres, España; e-mail: nando@unex.es

\begin{abstract}
RESUMEN
En este trabajo se revisan los resultados de 21 estudios experimentales sobre la regeneración de bosques de roble (Quercus spp.) en los que se analizan al menos tres de las fases del ciclo reproductivo: fertilización, desarrollo de frutos viables, dispersión, consumo postdispersivo, germinación-emergencia de plántulas, establecimiento de juveniles y reclutamiento de adultos. La mayoría de las poblaciones estudiadas se encontraban en bosques templados de Norteamérica y Europa, repartidos de forma equitativa entre bosques de ambientes xéricos (incluidos los mediterráneos) con especies perennes y habitualmente bajo explotación, y bosques de ambientes mésicos o montanos con especies generalmente caducifolias y escasamente alterados. Los estudios se clasificaron de acuerdo con los tipos de limitación de la regeneración encontrados por los autores, esto es, producción de semillas viables, presión de herbívoros y disponibilidad de sitios seguros. La revisión muestra que en la mayoría de los casos la regeneración se encontraba limitada por una combinación de factores, típicamente el consumo de bellotas y un ambiente abiótico desfavorable durante el establecimiento. La fecundidad de los árboles ha sido rara vez estudiada a pesar de que puede llegar ser en una limitación frecuente, al igual que ocurre con las limitaciones de la dispersión por animales. Finalmente, se analiza el grado de aplicación de la información ecológica acumulada a la solución de problemas de regeneración natural, y se discute su utilidad en comparación con las prácticas más comunes de regeneración artificial via plantaciones.
\end{abstract}

Palabras clave: dispersión, facilitación, herbivorismo, producción de semillas, Quercus, reclutamiento, regeneración forestal, robles, sitios seguros.

\begin{abstract}
This study presents a review of 21 experimental studies on oak (Quercus) regeneration analysing at least three phases of the reproductive cycle, namely fertilization, viable fruit production, acorn dispersal, postdispersal predation, seedling emergence, sapling establishment, and adult recruitment. Most study cases came from North American and European temperate forests representing a continuum from xeric sites with evergreen oaks (usually managed by humans) to mesic, well-preserved sites with deciduous oaks. Studies were classified according to three types of regeneration limitation, that is, viable seed production, herbivory, and safe sites availability. Results show that regeneration is usually limited by a combination of factors, tipically acorn consumption and unfavourable abiotic conditions for establishment. Tree fecundity, as well as seed dispersal by animals, are two important, usually neglected sources of recruitment limitation. On the other hand, anthropogenic disturbances negatively affects forest regeneration by changing the abundance or behavior of mutualistic or phytophagous organisms, and by modifying the abiotic scenario for seedling establishment. Finally, the application of ecological information for solving regeneration failures of oak forests is discussed, especially in relation to the alternatives of natural regeneration versus planting.
\end{abstract}

Key words: facilitation, forest regeneration, herbivory, oaks, Quercus, recruitment, safe sites, seed dispersal, seed limitation.

\section{INTRODUCCIÓN}

La regeneración de las plantas es un proceso dinámico por el que nuevos individuos se incorporan a la población reproductora a medida que otros desaparecen como resultado de la mortali- dad natural (Harper 1977). En el caso de las especies leñosas, este proceso implica una serie de transiciones entre estados (flores, semillas, plántulas, brinzales y adultos) que implican pérdidas en potencial reproductivo debidas a la actuación de factores abióticos y bióticos, cuyo 
efecto combinado determina el resultado final en número de nuevos individuos adultos (Jordano \& Herrera 1995). Entre los agentes bióticos implicados se encuentran al menos defoliadores, polinizadores, consumidores de semillas pre- y postdispersivos, diseminadores de semillas y ramoneadores, que participan en una compleja trama de interacciones cuyos efectos netos pueden ser en gran medida dependientes del escenario abiótico.

Los bosques de las zonas templadas se caracterizan por una diversidad comparativamente baja de especies arbóreas, siendo frecuentemente dominados por una sola especie (Walter 1985, Armesto et al. 1995). Así, la dinámica de la regeneración de estas especies condiciona en gran medida los procesos esenciales a nivel de ecosistema y la historia vital de los organismos que dependen de ellas (Jones et al. 1994). Esta es la razón por la que la falta de regeneración en las poblaciones de estas especies puede tener consecuencias muy significativas desde el punto de vista del mantenimiento de su capacidad productiva y de la conservación de los ecosistemas forestales y la diversidad biológica asociada (Armesto et al. 1995, Díaz et al. 1997, McPherson 1997, Perry 1998).

La deficiencia o ausencia de regeneración en una población de plantas puede ser consecuencia de una leve reducción en las probabilidades de transición entre varias fases del ciclo reproductivo o, más comúnmente, del colapso de alguna de sus fases (Herrera et al. 1994). La detección de estas fases críticas equivale en la práctica a determinar la contribución relativa de los agentes causantes de pérdidas en potencial reproductivo a lo largo del ciclo desde la floración al reclutamiento (ver Herrera et al. 1994, Pulido 1999, Cuevas 2000, Rey \& Alcántara 2000 para especies forestales). En el caso de los bosques sometidos a explotación, la incidencia de las prácticas forestales suele alterar profundamente los parámetros de los que depende la regeneración (Perry 1998). Así, por ejemplo, la disminución en la cobertura del dosel arbóreo tras la extracción de una parte de los árboles en bosques maderables supone una modificación significativa de las características microclimáticas, lo que normalmente implica cambios notables en las condiciones bióticas que condicionan la instalación de la regeneración (Barik et al. 1996, Buckley et al. 1998, Pulido et al. 2000 , entre otros). En el caso de sistemas silvopastorales los cambios en la densidad de los árboles remanentes y el aumento de la presión de los herbívoros afectan a la producción de semillas y a la abundancia y el comportamiento de los animales que interactúan con las especies arbóreas (ver revisiones en Díaz et al. 1997 y McPherson 1997).

Una gran parte de los bosques de la zona templada del hemisferio norte y de las transiciones subtropicales están dominados por especies del género Quercus ("robles" en sentido amplio). Este género contiene en torno a 400 especies, de las que la mayoría se encuentran en Norteamérica y algunas decenas en el área circunmediterránea y Asia oriental (Kaul 1985, Miller \& Lamb 1985). Gran parte de estos bosques han sido objeto de un aprovechamiento agroforestal que ha dado lugar a graves problemas de regeneración. Este es el caso de la mayoría de las sabanas de robles del oeste de Estados Unidos, los bosques de robles ingleses o las dehesas y montados de la Península Ibérica. En todos estos sistemas forestales se ha constatado que la transformación secular de los bosques nativos (principalmente para el aprovechamiento ganadero) es en gran medida incompatible con el establecimiento de plántulas y brinzales, lo que a la larga produce un envejecimiento irreversible del arbolado y, en algunas casos de deficiencia crónica en la regeneración, una disminución a escala geográfica de la superficie forestal (revisiones en Harmer 1994, McPherson 1997, varios autores en Pulido et al. en prensa).

Este trabajo constituye una revisión selectiva de la información ecológica existente sobre la regeneración de los robles, para lo que se sintetizan los resultados de aquellos trabajos que han abordado desde una perspectiva global el ciclo de regeneración. En él se plantean como objetivos básicos: (1) determinar la importancia relativa de los factores habitualmente citados como limitantes de la regeneración, esto es, la producción de semillas, la presión de herbívoros y la disponibilidad de sitios apropiados para el establecimiento; (2) discutir la utilidad potencial de esta información para el diseño de técnicas de restauración.

\section{HISTORIA NATURAL DE LA REPRODUCCIÓN EN QUERCUS}

Aunque algunos rasgos de la biología reproductiva del género difieren de unos subgéneros a otros (Kaul 1985), existe una considerable constancia geográfica en los parámetros morfológicos y fenológicos básicos, así como en la identidad de los gremios de animales que, a través de su interacción en distintas fases del ciclo, afectan al éxito reproductivo de los robles. Esta similitud permite la comparación de la dinámica de la regeneración en áreas muy distantes, que difieren básicamente 
en el escenario climático y en el grado de conservación. Seguidamente se resumen las características reproductivas necesarias para una comprensión de la dinámica de la regeneración, especialmente de los bosques norteamericanos y europeos, que han sido más intensamente investigados (véase Kaul 1985 y Vázquez 1998 para una descripción detallada).

En general, los robles son árboles de tamaño pequeño o mediano (3-20 m), perennes o caducifolios, monoicos y con polinización anemófila. Las inflorescencias masculinas y femeninas se producen en los brotes de crecimiento anual, emitidos entre febrero y abril en los bosques de la zona templada. Durante su elongación los brotes son defoliados principalmente por orugas de lepidópteros (típicamente polillas de las familias Tortricidae, Noctuidae y Lymantridae), que reducen la producción de frutos, bien por consumo directo de las flores femeninas o bien indirectamente, al reducir el porcentaje de frutos cuajados que se desarrollan hasta la madurez (Crawley 1985). Las flores femeninas que reciben polen son fertilizadas entre uno y tres meses después, siendo muy significativas las pérdidas por fallo en la fertilización, que suelen atribuirse a condiciones climáticas desfavorables (Wolgast \& Stout 1997, Pulido 1999). Con algunas excepciones entre los robles americanos, los frutos cuajados se desarrollan a lo largo del verano y principios del otoño, sufriendo considerables pérdidas antes de la dispersión debidas a distintas causas de aborto y la acción de insectos perforadores de los frutos (larvas de gorgojos y polillas). Una vez en el suelo, los frutos crecidos (bellotas) son consumidos por ungulados, roedores y aves. Algunas especies de roedores (géneros Apodemus, Peromyscus, Sciurus y Tamias principalmente) y aves de pequeño tamaño (notablemente los arrendajos de los géneros Garrulus, Aphelocoma y Cyanocitta), actúan como diseminadores al almacenar las bellotas a veces a una distancia considerable del árbol productor (véase Vázquez 1998, para una lista completa de consumidores y diseminadores). La germinación de las bellotas y la emergencia de plántulas ocurre en la primavera siguiente a la dispersión, existiendo una amplia variabilidad fenológica en función del clima y el hábito perenne o caducifolio (Fox 1982, Germaine \& McPherson 1998, Pulido 1999). Finalmente, en las fases de establecimiento y reclutamiento las plántulas y brinzales leñosos sufren la defoliación y el daño mecánico por parte de invertebrados y mamíferos ramoneadores, así como los efectos desfavorables de la sequía y la competencia del sotobosque.

\section{MATERIALES Y MÉTODOS}

\section{Criterios de selección}

Este trabajo revisa la información sobre la ecología de la regeneración de robledales. La búsqueda de información se ha limitado a las publicaciones científicas dentro del ámbito de la Ecología, decisión que se basa fundamentalmente en criterios de accesibilidad y adecuación del enfoque de los trabajos a las restricciones aquí planteadas. Así, se consideran sólo los trabajos publicados que abordan mediante experimentos mensurativos o manipulativos los procesos implicados en la regeneración. Como segunda restricción, se han considerado exclusivamente los trabajos que comprenden al menos tres de las siguientes fases del ciclo de regeneración (generalmente consecutivas): floración y producción de semillas, crecimiento y maduración de frutos, dispersión, consumo postdispersivo, germinación-emergencia de plántulas, establecimiento de brinzales y reclutamiento de adultos (véase Jordano \& Herrera 1995). Este planteamiento garantiza que los estudios individuales puedan ser asignados a categorías de limitaciones de la regeneración previamente definidas (producción de semillas, consumo por herbívoros y disponibilidad sitios seguros), y que como resultado de la revisión se obtenga una visión concluyente de su importancia relativa (Clark et al. 1999). Además, en términos de transferencia de la información al ámbito aplicado de la conservación, este procedimiento aporta información más útil para la toma de decisiones que el análisis individualizado de la información descriptiva acumulada para cada una de las etapas del ciclo.

Para la elaboración de la base de datos en que se apoya este trabajo, se han clasificado los artículos que se ajustaban a las condiciones citadas según dos grupos de criterios. Por un lado, se ha considerado la localización y el contexto ambiental y de aprovechamiento en que se encontraba la población estudiada (Tabla 1). Por otro, se ha asignado a cada artículo la causa o causas de limitación de la regeneración indicada por el autor (Tabla 2). Cuando esta indicación no se hacía explícitamente en el trabajo, se ha considerado que un factor era limitante cuando cabe deducir de la investigación un incremento de la regeneración en respuesta a una eventual supresión o atenuación de los efectos del factor (e.g., Crawley \& Long 1995, Turnbull et al. 2000). Finalmente, cuando un mismo artículo presentaba resultados de dos o más localidades de estudio suficientemente distantes y sometidas a condiciones claramente diferenciadas (e.g., pre- 
PULIDO

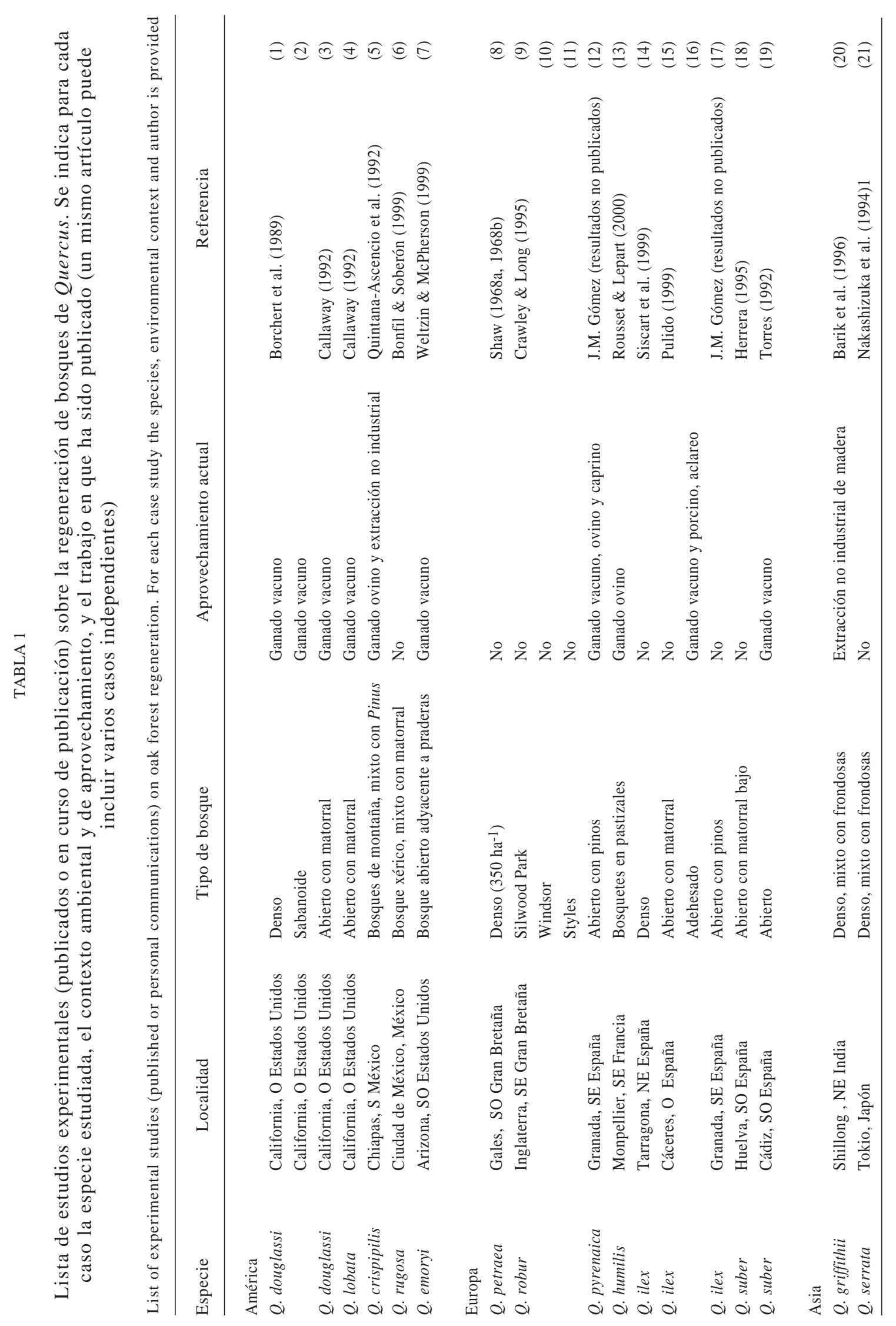


sencia/ausencia de pastoreo), los resultados de cada sitio se han considerado como casos independientes.

\section{RESULTADOS}

\section{Tendencias en la dinámica de la regeneración}

El número de artículos publicados que se ajusta a los criterios citados es de 16 , e incluyen un total de 21 casos de poblaciones independientes analizadas. Según la procedencia geográfica, la mayoría de los casos $(90 \%)$ se han estudiado en Europa (12) y América (siete), con tan sólo dos trabajos documentados en Asia oriental. En cuanto al escenario climático, se ha encontrado un reparto equitativo entre los ambientes xéricos (sitios de baja productividad, semiáridos o mediterráneos y con especies perennes; $52 \%$ de casos) y mésicos ( sitios de alta productividad y/o en áreas de montaña con especies generalmente caducifolias; 48 $\%$ de casos).

En cuanto a la profundidad de los estudios, equivalente aquí al número de transiciones del ciclo de regeneración analizadas, un $71 \%$ de los casos se refieren a análisis "parciales" (tres fases analizadas) y abordan casi siempre el consumo postdispersivo, la germinación y el establecimiento (Tabla 2). Sólo se dispone de seis $(29 \%)$ casos de estudios "globales" (que analizan las fases pre- y postdispersiva), dentro de tres estudios con las especies europeas $Q$. petraea, $Q$. robur y $Q$. ilex. Estas dos últimas especies son, junto con la norteamericana $Q$. douglassi, las más frecuentemente investigadas.

A pesar de que el número de casos identificados es insuficiente para un análisis estadístico detallado, las restricciones impuestas en la revisión hacen que en general los estudios que cumplen los criterios de selección sean concluyentes y puedan ser asignados a uno o más de los tipos de limitación definidos. Así, siete (33 \%) de los casos presentan evidencia de un solo tipo de limitación, mientras que $14(67 \%)$ sugieren la existencia de múltiples limitaciones de la regeneración. Dado que la mayoría de los trabajos no abordan en el análisis las tres limitaciones descritas, hay que considerar como conservativa la cifra referida a limitaciones múltiples. La combinación de factores limitantes, que parece ser la norma en los estudios analizados, implica típicamente la presión de consumidores de semillas y factores microclimáticos desfavorables para el establecimiento.
Importancia relativa de las limitaciones a la regeneración

Seguidamente se evalúa el nivel de conocimientos adquiridos y el peso relativo de cada uno de los tipos de limitación. Aunque esta discusión se articula en epígrafes independientes, es preciso tener en cuenta que la limitaciones definidas pueden no ser independientes, por lo que también es preciso considerar la interacción entre los efectos de los factores limitantes.

Producción de semillas. La importancia de las limitaciones impuestas por la fecundidad para la regeneración de los robles ha sido probablemente infravalorada como consecuencia del sesgo metodológico que supone centrarse en las fases postdispersivas. Esta decisión es justificada a posteriori en la mayoría de los estudios, una vez comprobado que los resultados en término de establecimiento de plántulas no difieren en años de alta y baja producción de semillas. No obstante, cuatro de los siete casos en los que se analiza la influencia de este factor sugieren la existencia de efectos limitantes, especialmente el estudio de Crawley \& Long (1995), en el que se documenta una estrecha asociación entre picos de semillazón y establecimiento de brinzales a lo largo de 15 años.

Para comprender el significado de estas limitaciones es preciso contextualizar el análisis considerando la longevidad de las especies de robles y la relación de la fecundidad con otros factores. Así, por una parte, el reclutamiento de los robles parece estar limitado por la fecundidad sólo en años malos pero se dispara en los ocasionales años de semillazón, lo que, según algunos autores, se relacionaría con una estrategia de saciado de consumidores (Sork et al. 1993, Crawley \& Long 1995, entre otros). Este reclutamiento exitoso en algunos años puede ser suficiente para mantener una estructura de edades estable sin que se produzcan deficiencias en la regeneración. Por otra parte, el predominio de años de baja producción es un fenómeno que, aunque no generalizable, se observa muy a menudo en los robledales sin que ello implique bajos niveles de reclutamiento. La ausencia de efectos demográficos de esta baja fecundidad se explica por la existencia de limitaciones posteriores, generalmente la falta de sitios seguros para el establecimiento (véase Andersen 1989 para una discusión general, y Crawley \& Long 1995, para un ejemplo con $Q$. robur).

Presión de herbívoros. Quizá el resultado más común de los estudios que analizan el consumo de semillas en la fase postdispersiva de la regeneración de los robledales es la desaparición total o casi de las bellotas, especialmente si están con- 


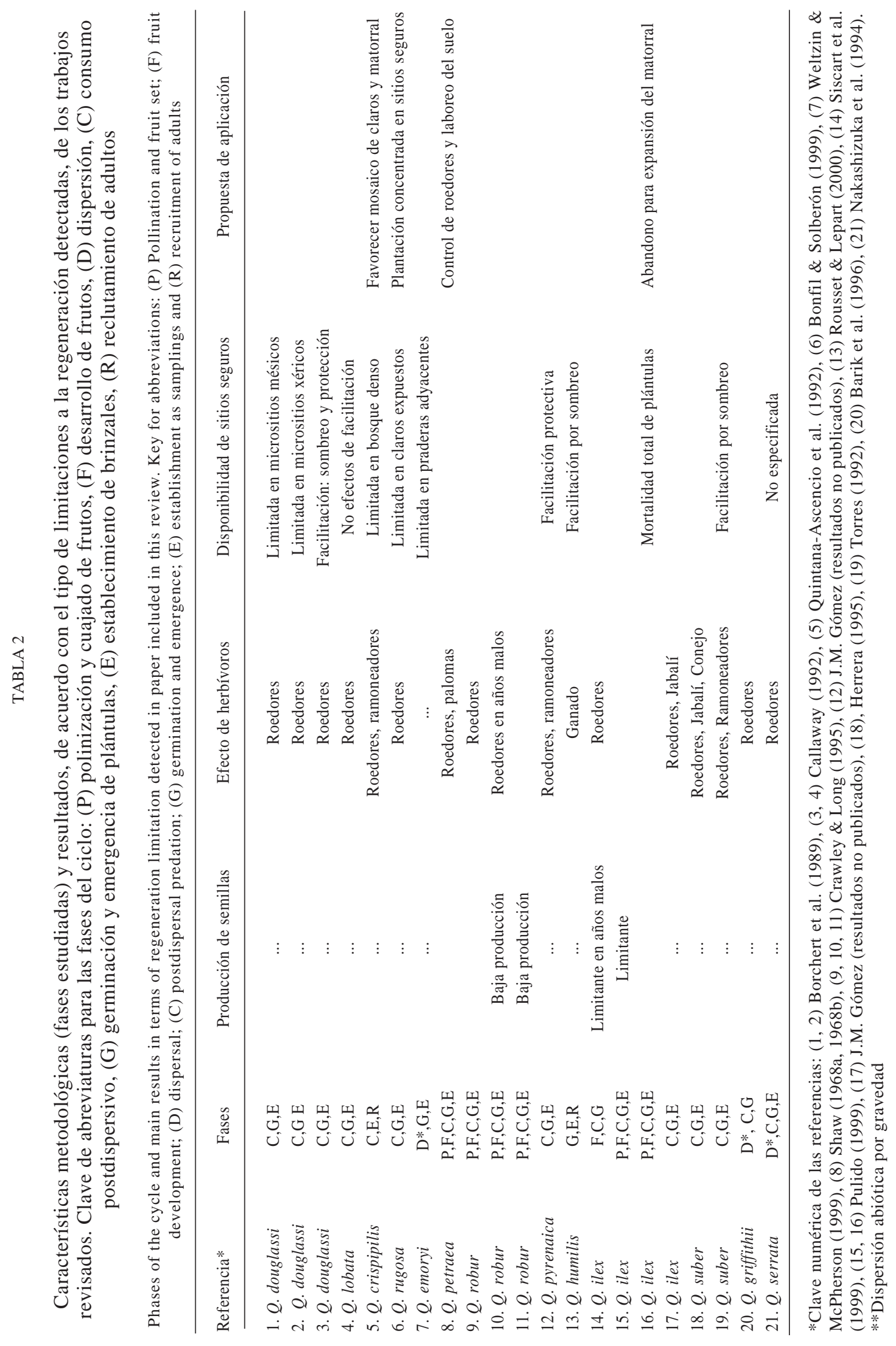


centradas de forma natural bajo los árboles (Herrera 1995, Barik et al. 1996, Pulido 1999). Cuando las bellotas son ubicadas experimentalmente lejos de los árboles y de forma más dispersa para analizar el efecto del microhábitat sobre el consumo, los resultados muestran que el riesgo aumenta en áreas de matorral, donde los roedores concentran su actividad. De hecho, estos animales figuran como la principal causa de mortalidad postdispersiva de bellotas en el $76 \%$ de los casos estudiados (Tabla 2), si bien su acción se combina casi siempre con la de los ungulados y nunca se ha demostrado que sean el único factor limitante (Boucher 1981, Herrera 1995, Santos \& Tellería 1997, Pulido 1999, J.M. Gómez resultados no publicados). Por otra parte, la interferencia de los ungulados limita tanto el número de bellotas dispersadas por roedores como el establecimiento de plántulas, al buscar las bellotas lejos de los árboles con mayor intensidad en torno a madrigueras y almacenes de roedores (Focardi et al. 2000, ver además Pulido 1999, J.M. Gómez resultados no publicados para la interacción entre jabalíes, roedores y $Q$. ilex).

La mortalidad de plántulas y brinzales de roble es atribuible generalmente a mamíferos medianos (grandes roedores o lagomorfos) y a ungulados salvajes o domésticos. Las limitaciones a la regeneración encontradas en esta fase son cuantitativamente escasas (Tabla 2), aunque ello puede tener una explicación puramente metodológica. Por una parte, estos efectos sólo se manifiestan una vez superados el consumo de semillas por animales y los efectos abióticos durante el primer año, dos filtros generalmente severos que no dejan lugar a la actuación ulterior de otros factores. Por otra parte, la escala de tiempo necesaria para analizar el efecto del ramoneo sobre el reclutamiento excede en gran medida la duración de los estudios experimentales de la dinámica de la regeneración. Dado que la mayoría de los animales ramoneadores son además consumidores de bellotas, sus efectos globales pueden exceder cuantitativamente el de los roedores en situaciones concretas (29\% de los estudios en la Tabla 2). En cualquier caso, más que una limitación general a la regeneración, la acción de los ungulados supone una modificación del patrón de reclutamiento debido a la especifidad espacial del consumo de semillas y el ramoneo (J.M. Gómez resultados no publicados para $Q$. ilex y $Q$. pyrenaica).

Disponibilidad de sitios seguros. Aunque la adecuación de un micrositio para la regeneración puede manifestarse en la fase de semilla, plántula o brinzal (Schupp 1995), en este trabajo se restringe el concepto de sitio seguro a las dos últimas fases y especialmente a los efectos microclimáticos y competitivos específicos de una ubicación determinada dentro de un paisaje heterogéneo. Como demuestran varios estudios con robles (Tabla 2), estos efectos son altamente dependientes del contexto incluso para una misma especie. Así, la regeneración de $Q$. douglasii en California se ve favorecida en claros de localidades montanas y en áreas sombreadas de zonas bajas (Borchert et al. 1989). A nivel interespecífico, el rango de tolerancia a la sombra es un buen factor discriminante de las posibilidades de regeneración, hasta el punto de que las especies caducifolioas de sitios mésicos prosperan en claros naturales o antropogénicos (QuintanaAscencio et al. 1992, Buckley et al. 1998) y las perennes de sitios xéricos lo hacen sólo en áreas sombreadas (Callaway 1992, Torres 1992, Bonfil \& Soberón 1999, Pulido 1999, Weltzin \& McPherson 1999, Rousset \& Lepart 2000).

En bosques abiertos de ambientes mediterráneos la presencia de un estrato arbustivo de especies pioneras es una fuente de heterogeneidad añadida de gran importancia para la regeneración. Como ya se ha dicho, estas áreas arbustivas concentran el consumo por roedores, pero este efecto parece compensarse por la facilitación que suponen el sombreo y la protección ante los ramoneadores (Tabla 2). Así lo sugieren las frecuentes situaciones en que las investigaciones encuentran una elevada pérdida de bellotas experimentales y una mayor concentración de plántulas y brinzales bajo los arbustos (Rousset \& Lepart 2000, J.M. Gómez resultados no publicados, ver también Callaway 1992, Hulme 1996).

Además de la facilitación (microclimática o protectora), la asociación con arbustos o herbáceas implica efectos competitivos por la luz, el agua y los nutrientes. Dos estudios recientes (Buckley et al. 1998 con Q. rubra en bosques mésicos y Rousseau \& Lepart 2000 con Q. humilis en el Mediterráneo) sugieren que los efectos de interferencia (intercepción de la luz, o el agua y los nutrientes) se ven compensados por el de la facilitación (sombreo o protección). En otras palabras, los efectos negativos de la presencia de sotobosque parecen manifestarse solamente en términos de reducción del crecimiento, mientras que los efectos negativos de la ausencia de sotobosque acarrean normalmente una mortalidad acentuada. No obstante, es necesario profundizar en el estudio de estas relaciones extendiéndolo a un mayor número de situaciones de estrés ambiental y capacidad competitiva de las especies implicadas. 
Una limitación más: dispersión de semillas

De los 21 casos estudiados que se recogen en la Tabla 2, sólo dos hacen mención específica del papel limitante que la dispersión de las bellotas puede jugar en la regeneración (Weltzin \& McPherson 1999, ver también Hubbard \& McPherson 1999). Las bellotas se desprenden por gravedad una vez alcanzada la madurez, en un proceso que rara vez distancia las bellotas del árbol productor, a no ser en bosques que crecen en laderas de cierta pendiente. Aún en este caso las bellotas suelen ser consumidas con una probabilidad parecida a las que caen bajo la copa. Por el contrario, el carácter direccional de la dispersión secundaria por animales garantiza un mayor alejamiento de la planta madre y la deposición en pequeños almacenes, lo que disminuye el riesgo de consumo y desecación (Shaw 1968a, Barik et al. 1996). Este proceso de zoocoría parece, no obstante, haber sido ignorado repetidamente en los estudios de la regeneración de los robledales, seguramente por la dificultad metodológica del seguimiento de las bellotas dispersadas.

Según se deduce de los estudios disponibles, la limitación por la falta de dispersión es más probable en bosques abiertos de sitios xéricos, donde sus efectos se combinan con la falta de sitios seguros (sombreados y protegidos) para el establecimiento. Por contra, en bosques densos donde no existe la limitación de sombra y existe una capa protectora de hojarasca, la dispersión puramente abiótica garantiza la llegada de semillas a los abundantes micrositios seguros, sin que sea necesaria la participación de vectores especializados (Pulido \& Díaz resultados no publicados). En síntesis, la dispersión direccional tenderá a ser más necesaria cuanto menor sea la disponibilidad de sitios seguros y mayor la preferencia de los diseminadores por ellos. En ambientes secos, los efectos de la facilitación de los arbustos sólo se harán efectivos si los animales concentran las semillas dispersadas en estos micrositios, lo que otorga a la dispersión un papel demográfico clave (Herrera 1995, Hubbard \& McPherson 1999, Weltzin \& McPherson 1999, Pulido 1999).

\section{REGENERACIÓN Y CONSERVACIÓN}

\section{Influencia de la intervención humana}

Las proporciones de sitios con y sin aprovechamiento ganadero o forestal actual estuvieron desviadas hacia los primeros (57 y $43 \%$, respectivamente). Además, la frecuencia de aprovechamiento es significativamente mayor entre los bosques de ambientes xéricos y especies perennes (prueba de Chi-cuadrado, $\chi^{2}=4,49$, g.1. $\left.=1, P=0,034\right)$. En los estudios realizados en estos ambientes la necesidad de la investigación se justificaba invariablemente por un fallo en la regeneración previamente reconocido en una estructura de edades inestable y carente de individuos jóvenes (Tabla 2, Callaway \& Davis 1998, Pulido et al. 2001). En ambientes más productivos, sólo dos estudios consideraban a priori la existencia de este problema (Shaw 1968a, 1968b, Barik et al. 1996), si bien los robledales británicos, debido a su tradicional explotación ganadera y forestal, han sido objeto de preocupación por su falta de regeneración desde principios del siglo XX (Harmer 1994).

La asociación descrita entre aprovechamiento y problemas de regeneración ha de explicarse atendiendo a los efectos que la actividad humana ocasiona sobre aquellos rasgos ambientales de los que depende la regeneración. Independientemente de la situación original, el aprovechamiento da lugar a bosques más abiertos por extracción selectiva de árboles, situación que además suele mantenerse mediante la presión del pastoreo. En estas circunstancias es de esperar que los efectos del aprovechamiento sobre la regeneración sean mayores en bosques sometidos a estrés ambiental (Bertnees \& Callaway 1994), típicamente una combinación de suelos pobres, altas temperaturas y sequías pronunciadas. La simplificación estructural del bosque implica la desaparición de sitios seguros y, en algunos casos, cambios en la abundancia y comportamiento de animales mutualistas (diseminadores) o enemigos naturales (ganado; Barik et al. 1996, Pulido 1999, Rousseau \& Lepart 2000, J.M. Gómez resultados no publicados).

En bosques de sitios productivos se han documentado efectos positivos de la intervención humana sobre el éxito de alguna de las fases de la regeneración, y, de hecho, se han desarrollado algunas prácticas silviculturales para favorecer la regeneración natural (Harmer 1994). Es bien conocido, por ejemplo, que la extracción de parte de los árboles aumenta la fecundidad de los individuos remanentes en robledales templados (Shaw 1969a), mediterráneos (Pulido 1999) y subtropicales (Barik et al. 1996). Por otra parte, el aclareo moderado del bosque puede favorecer el establecimiento de brinzales, como se ha observado en $Q$. crispipilis (una especie intolerante de la sombra) en Chiapas en situaciones con escasa presión de herbívoros (Quintana-Ascencio et al. 1992). No obstante, ninguno de los trabajos revisados sugiere que estos incrementos parciales del éxito reproductivo den lugar a un correspondiente aumento de la tasa de regeneración. 
Utilidad de la información y aplicación en la restauración forestal

En la Tabla 2 se especifican aquellos trabajos que reconocían en su planteamiento la existencia de un problema de regeneración y que incluyen en sus aportaciones alguna recomendación práctica para solucionarlo. Si bien la mayoría de los trabajos publicados justificaban su interés en la utilidad aplicada de los posibles resultados, sólo cuatro $(25 \%)$ exponen recomendaciones prácticas. Estos estudios se refieren a los bosques alterados del centro y sur de México, los robledales británicos y las dehesas (pastizales arbolados similares a las sabanas) del oeste de España. Resulta llamativa la falta de alusiones en los trabajos sobre las sabanas del oeste de Estados Unidos, que sufren problemas serios de regeneración que han sido muy investigados. Esta falta de transferencia de resultados científicos a medidas prácticas no es del todo atribuible a la vocación básica de los ecólogos que han abordado la dinámica de la regeneración. Más probablemente, este hecho puede atribuirse al uso extendido de las plantaciones artificiales como forma de combatir la falta de regeneración natural. Estas prácticas de reforestación han sido muy utilizadas en Norteamérica (Standiford 1991) y Europa (Harmer 1994), donde la realización de costosas inversiones generalmente subsidiadas garantiza el éxito de los programas de reforestación.

Aparentemente los conocimientos ecológicos acumulados sobre la regeneración de bosques de robles no ha servido para promover la alternativa de la regeneración natural. Sencillamente, en opinión de los selvicultores, la plantación ahorra incertidumbres económicos frente a los resultados difícilmente predecibles de la regeneración natural, pudiendo ser además compatible con los usos de la tierra anteriores a la actuación (Harmer 1994). A pesar de que esta filosofía pudiera justificarse cuando el objetivo de la regeneración es la producción forestal, los métodos alternativos de restauración natural pueden ser útiles en sistemas de uso múltiple o en áreas con un alto valor de conservación donde se prime la integridad ecológica de los sistemas protegidos. Las técnicas de regeneración natural son bien conocidas en centroeuropa e Islas Británicas (Harmer 1994, Larsen \& Jonson 1998, Von Lupke 1998), donde han sido preferentemente aplicadas en bosques con especial significado cultural o de conservación.

En áreas de bosque mediterráneo, por el contrario, estas técnicas son aplicables en amplias zonas de montaña o llanura donde la ganadería y otras prácticas asociadas han impedido la regene- ración del bosque (Jordano et al. 2000, Pulido et al. 2001, J.M. Gómez resultados no publicados). Se da además la circunstancia de que estas propuestas de regeneración natural han venido precedidas de estudios sobre ecología de la regeneración natural acompañados de análisis indicadores de los menores costes económicos y ambientales en comparación con técnicas tradicionales de plantación (Castro et al. 2001, Pulido et al. en prensa). Así, para sistemas silvopastorales de dehesa (Díaz et al. 1997), se ha detectado un déficit generalizado de regeneración por la ausencia de áreas de matorral a las que se asocian los animales diseminadores y que proporcionan sitios seguros ante el riesgo de ramoneo y de desecación de las plántulas en el primer verano (Pulido 1999). La ausencia de matorral es debida a las intervenciones de aclareo iniciales y se mantiene mediante una elevada presión de pastoreo por el ganado. Así, un abandono temporal (10-20 años) de la actividad ganadera en una parte del territorio gestionado permitiría la invasión de especies pioneras del matorral, con lo que se propicia la dispersión y la facilitación (Pulido 1999). Las "manchas" de matorral, además de ayudar a la regeneración del arbolado, son utilizadas dentro de un sistema de aprovechamiento cinegético que se ha mostrado compatible con los usos puramente ganaderos o forestales de la dehesas y con la preservación de la diversidad biológica de estos sistemas (Díaz et al. 1997).

De forma similar, los bosques de montaña del sureste de España, muy degradados por las prácticas antrópicas, deben su déficit de regeneración a la falta de matorral facilitador de la supervivencia de propágulos de varias especies arbóreas dominantes o amenazadas (Jordano et al. 2000, Castro et al. 2001, J.M. Gómez resultados no publicados). Las técnicas tradicionales de plantación, muy agresivas en estas zonas de suelo degradado y elevada pendiente, han demostrado ser menos eficientes que una nueva técnica basada en la siembra o plantación dirigida bajo arbustos facilitadores (Castro et al. 2001). Estos dos casos constituyen ejemplos interesantes de cooperación científico-técnica que están demostrando su utilidad práctica en la restauración de áreas de valor tanto ambiental como productivo.

\section{AGRADECIMIENTOS}

El autor agradece a Anna Traveset la invitación a participar en el Taller Iberoamericano sobre "Aspectos teóricos de la biología reproductiva de plantas y su relevancia para la conservación" (Mallorca, octubre de 2000), y a Mary Kalin- 
Arroyo su trabajo como coordinadora en la Red Iberoamericana de Ecosistemas Mediterráneos en que se enmarcó el taller. La convivencia y las discusiones con los participantes han sido un estímulo decisivo para la conclusión de este trabajo.

\section{LITERATURA CITADA}

ANDERSEN AN (1989) How important is seed predation to recruitment in stable populations of long-lived perennials? Oecologia 81: 310-315.

ARMESTO JJ, C VILLARÁN \& MK ARROYO (eds) (1995) Ecología de los bosques nativos de Chile. Editorial Universitaria, Santiago, Chile. 477 pp.

BARIK SK, RS TRIPATHI, HN PANDEY \& P RAO (1996) Tree regeneration in a subtropical humid forest: effect of cultural disturbance on seed production, dispersal and germination. Journal of Applied Ecology 33: 1551-1560.

BERTNESS MD \& RM CALLAWAY (1994) Positive interactions in communities. Trends in Ecology and Evolution 9: 191-193.

BONFIL C \& J SOBERÓN (1999) Quercus rugosa seedling dynamics in relation to its re-introduction in a disturbed Mexican landscape. Applied Vegetation Science 2: 189-200.

BORCHERT MI, FW DAVIS, J MICHAELSEN \& LD OYLER (1989) Interaction of factors affecting seedling recruitment of blue oaks (Quercus douglasii) in California. Ecology 70: 389-404.

BOUCHER DH (1981) Seed predation by mammals and forest dominance by Quercus oleoides, a tropical lowland oak. Oecologia 49: 409-414.

BUCKLEY DS, TL SHARIK \& JG ISEBRANDS (1998) Regeneration of norther red oak: positive and negative effects of competitor removal. Ecology 79: 65-78.

CALLAWAY R (1992) Effect of shrubs on recruitment of Quercus douglassi and Quercus lobata in California. Ecology 73: 2118-2128.

CALLAWAY RM \& FW DAVIS (1998) Recruitment of Quercus agrifolia in central California: the importance of shrub-dominated patches. Journal of Vegetation Science 9: 647-656.

CASTRO J, R ZAMORA, JA HÓDAR \& JM GÓMEZ (en prensa) The use of shrubs as nurse plants: a new technique for reforestation in Mediterranean mountains. Restoration Ecology.

CLARK JS, B BECKAGE, P CAMILL, B CLEVELAND, J LAMBERS, J LICHTER, J MACLACHLAN, J MOHAN \& P WYCOFF (1999) Interpreting recruitment limitation in forests. American Journal of Botany 86: 1-16.

CRAWLEY MJ (1985) Reduction of oak fecundity by lowdensity herbivore populations. Nature 314: 163-164.

CRAWLEY MJ \& CR LONG (1995) Alternate bearing, predator satiation and seedling recruitment in Quercus robur L. Journal of Ecology 83: 683-696.

CROW TR (1988) Reproductive mode and mechanisms of self-replacement of Northern red oak (Quercus rubra): a review. Forest Science 34: 19-40.
DÍAZ M, P CAMPOS \& FJ PULIDO (1997) The Spanish dehesa: a diversity in land-use and wildlife. En: DJ Pain \& MW Pienkowsk (eds) Farming and birds in Europe: 178-209. Academic Press, London, England.

FOX JF (1982) Adaptation of grey squirrel behaviour to autumn germination of white oak acorns. Evolution 36: 800-809.

HARMER R (1994) Natural regeneration of broadleaved trees in Britain. I. Historical Aspects. Forestry 67: 179-188.

HARPER JL (1977) Population biology of plants. Academic Press, London, England. 892 pp.

HERRERA CM, JORDANO P, LÓPEZ-SORIA L, AMAT JA (1994) Recruitment of a mast-fruiting, birddispersed tree: bridging frugivore activity and seedling establishment. Ecological Monographs 64: 315-344.

HERRERA J (1995) Acorn predation and seedling production in a low-density population of cork oak (Quercus suber L.). Forest Ecology and Management 76: 197-201.

HULME PH (1996) Natural regeneration of yew (Taxus baccata L.): microsite, seed or herbivore limitation? Journal of Ecology 84: 853-861.

JONES CG, JH LAWTON \& M SCHACHAK (1994) Organisms as ecosystem engineers. Oikos 69: 373386.

JORDANO P \& HERRERA CM (1995) Shuffling the offspring: uncoupling and spatial discordance of multiple stages in vertebrate seed dispersal. Écoscience 2: 230-237.

JORDANO P, R ZAMORA, T MARAÑÓN \& J ARROYO (en prensa) Ecological and demographic research in Mediterranean forests of southern Spain: applications to conservation and restoration. Proceedings of the Symposium of Forest Research, Tesalónica, Grecia.

LARSEN DR \& PS JOHNSON (1998) Linking the ecology of natural regeneration to silviculture. Forest Ecology and Management 106: 1-7.

McPHERSON GH (1997) Ecology and management of North American savannas. Universidad de Arizona, Tucson, Arizona. 208 pp.

MILLER HA \& SH LAMB (1985) Oaks of Noth America. Naturegraph Publishers, Happy Camp, California. 327 pp.

NAKASHIZUKA T, S IIDA, T MASAKI, M SHIBATA \& H TANAKA (1995) Evaluating increased fitness through dispersal: a comparative study on tree populations in a temperate forest, Japan. Écoscience 2: $245-251$

PERRY DA (1998) The scientific basis of forestry. Annual Review of Ecology and Systematics 29: 435-466.

PULIDO FJ (1999) Herbivorismo y regeneración de la encina (Quercus ilex L.) en bosques y dehesas. Tesis Doctoral, Universdad de Extremadura, Cáceres, España. $146 \mathrm{pp}$

PULIDO FJ, B DÍAZ \& G MARTÍNEZ-PASTUR (2000) Incidencia del ramoneo del guanaco (Lama guanicoe) sobre la regeneración temprana de lenga (Nothofagus pumilio) en Tierra del Fuego, Argentina. Investigación Agraria, Sistemas y Recursos Forestales (Argentina) 9: 381-394. 
PULIDO FJ, M DÍAZ \& SJ HIDALGO (2001) Sizestructure and regeneration of holm oak (Quercus ilex) forests and dehesas: effects of agroforestry use on their long-term sustainability. Forest Ecology and Management 146: 1-13.

PULIDO FJ, P CAMPOS \& G MONTERO (eds) (2002) La gestión forestal de las dehesas. Instituto del Corcho, la Madera y el Carbón, Mérida, España.

QUINTANA-ASCENCIO PF, M GONZÁLEZ-ESPINOSA \& N RAMÍREZ-MARCIAL (1992) Acorn removal, seedling survivorship, and seedling growth of Quercus crispipilis in successsional forest of the highland of Chiapas, Mexico. Bulletin of the Torrey Botanical Club 119: 6-18.

REY PJ \& J ALCÁNTARA (2000) Recruitment dynamics of a fleshy-fruited plant (Olea europarea): connecting patterns of seed dispersal to seedling establishment. Journal of Ecology 88: 622-633.

ROUSSEAU O \& J LEPART (2000) Positive and negative interactions at different stages of a colonizing species (Quercus humilis). Journal of Ecology 88: 401-412.

SANTOS T \& JL TELLERÍA (1997) Vertebrate predation on Holm oak, Quercus ilex, acorns in a fragmented habitat: effects on seedling recruitment. Forest Ecology and Management 98: 181-187.

SCHUPP EW (1995) Seed-seedling conflict, habitat choice, and patterns of plant recruitment. American Journal of Botany 82: 399-409.

SHAW MW (1968a) Factors affecting the natural regeneration of sessile oak (Quercus petraea) in north Wales. I. A preliminary study of acorn production, viability and losses. Journal of Ecology 56: 565-583.

SHAW MW (1968b) Factors affecting the natural regeneration of sessile oak (Quercus petraea) in north Wales. II. Acorn losses and germination under field conditions. Journal of Ecology 56: 647-661.
SISCART D, V DIEGO \& F LLORET (1999) Acorn ecology. En: Rodá F, C Gracia, J Retana \& J Bellot (eds) The ecology of Mediterranean evergreen oak forests: 75-87. Springer-Verlag, Berlin, Germany.

SORK VL, J BRAMBLE \& O SEXTON (1993) Ecology of mast fruiting in three species of North American decidious oaks. Ecology 74: 528-541.

STANDIFORD R (ed) (1991) Proceedings of the symposium on oak woodland and hardwood rangeland management. Pacific Southwest Research Station, Berkeley, California. 376 pp.

TURNBULL L, MJ CRAWLEY \& M REES (2000) Are plant populations seed-limited? a review of seed sowing experiments. Oikos 88: 225-238.

VÁZQUEZ FM (1998) Semillas de Quercus: biología, ecología y manejo. Consejería de Agricultura y Comercio, Badajoz, España. 234 pp.

VON LUPKE B (1998) Silvicultural methods of oak regeneration with especial respect to shade tolerant mixed species. Forest Ecology and Management 106: 19-26.

WALTER H (1985) Vegetation of the earth. SpringerVerlag, Berlin, Germany. 318 pp.

WELTZIN JF \& GR MCPHERSON (1999) Facilitation of conespecific seedling recruitment and shifts in temperate savanna ecotones. Ecological Monographs 69: 513-534

WOLGAST LJ \& BB STOUT (1997) The effects of relative humidity at the time of flowering on fruit set in bear oak (Quercus ilicifolia). American Journal of Botany 64: 159-160.

Editor Asociado: R. Medel

Recibido el 30 de abril de 2001; aceptado el 8 de enero de 2002 